\title{
Interactions of human tenascin-X domains with dermal extracellular matrix molecules
}

\author{
David Egging • Franka van den Berkmortel • \\ Glen Taylor · Jim Bristow • Joost Schalkwijk
}

Received: 5 July 2006 / Revised: 28 August 2006 / Accepted: 2 September 2006 / Published online: 11 October 2006

(C) Springer-Verlag 2006

\begin{abstract}
Tenascin-X (TNX) is a large $450 \mathrm{kDa}$ extracellular matrix protein expressed in a variety of tissues including skin, joints and blood vessels. Deficiency of TNX causes a recessive form of Ehlers-Danlos syndrome characterized by joint hypermobility, skin fragility and hyperextensible skin. Skin of TNX deficient patients shows abnormal elastic fibers and reduced collagen deposition. The mechanism by which TNX deficiency leads to connective tissue alterations is unknown. Here we report that C-terminal domains of human TNX bind to major dermal fibrillar collagens and tropoelastin. We have mapped these interactions to the fibronectin type III repeat 29 (FNIII29) and the C-terminal fibrinogen domain $(\mathrm{FbgX})$ of TNX. In addition we found that FNIII29 of TNX accelerates collagen fibrillogenesis in vitro. We hypothesize that TNX contributes to matrix stability and is possibly involved in collagen fibril formation.
\end{abstract}

Electronic supplementary material Supplementary material is available in the online version of this article at http://dx.doi.org/ 10.1007/s00403-006-0706-9 and is accessible for authorized users.

D. Egging $(\varangle) \cdot$ F. van den Berkmortel $\cdot$ J. Schalkwijk Department of Dermatology, Nijmegen Centre for Molecular Life Sciences, Radboud University Nijmegen Medical Centre, P.O. Box 9101, 6500 HB Nijmegen, The Netherlands e-mail: d.egging@derma.umcn.nl

G. Taylor $\cdot$ J. Bristow

Department of Pediatrics, University of California, San Francisco, CA, USA

\section{J. Bristow}

Department of Genome Sciences, Lawrence Berkeley National Laboratory,

Berkeley, CA, USA
Keywords Tenascin-X Collagen · Elastin · Fibrillogenesis $\cdot$ Ehlers-Danlos syndrome

\section{Introduction}

Tenascin- $\mathrm{X}$ is a large extracellular matrix glycoprotein composed of EGF like-repeats, fibronectin type III (FNIII) repeats and a C-terminal fibrinogen domain (FbgX) [1, 5, 6, 12]. TNX abnormalities are associated with several pathological conditions [2, 22, 27]. Complete deficiency of TNX in humans leads to a recessive form of Ehlers Danlos syndrome (EDS) and TNX haploinsufficiency is a cause of hypermobility type EDS. The skin of TNX deficient patients is markedly lax with poor recoil properties and shows easy bruising [2, 14, $20,22,27]$. We have previously shown TNX to be present in the entire dermis of healthy individuals. During development in mice TNX colocalises with the major fibrillar collagens and elastic fibers in the dermis [3,22]. Adult TNX deficient patients show abnormal elastic fibers and reduced collagen deposition in skin [28]. The mechanism by which TNX deficiency leads to alterations in the extracellular matrix (ECM) is unknown. Mapping of TNX domains that interact with components of the elastic fibers or collagen is therefore an important step to define the role of TNX in connective tissue biology of human skin.

It has previously been shown that bovine and murine TNX bind to heparin $[13,16]$. In bovine TNX a conformational heparin-binding site has been identified involving FNIII repeats 10 and 11 in the N-terminal half of the molecule. This heparin-binding site is also involved in the binding of TNX to decorin, and it was proposed that TNX interacts with collagen through 
decorin [4]. Minamitani et al. showed that the short isoform of murine TNX binds directly to collagen type I, probably through interaction with one of the fibronectin type III repeats. This short isoform was found to promote collagen fibrillogenesis in vitro [18].

The aim of our study was to investigate the molecular mechanisms of the observed phenotype in TNX-deficient EDS patients. We therefore analyzed interactions of human TNX with elastic fiber components and with the major fibrillar collagens in the dermis (collagen type I, III and V). We found that the C-terminus of TNX harbors potential interaction sites with collagens type I, III and V and tropoelastin (TE), which are constituents of the anatomical structures that are abnormal in the dermis of these patients.

\section{Materials and methods}

Expression and purification of recombinant TNX fragments

The TNX fragments were amplified by PCR with the primers listed in Table 1 using a previously described $2.7 \mathrm{~kb}$ human TNX cDNA [19] as a template. PCR products were ligated into the $\mathrm{pCR} 2.1 \mathrm{TOPO}$ vector (Invitrogen, Breda, NL) according to the manufacturer's instructions for easy digestion with restriction enzymes. The pCR2.1 TOPO vectors with inserts were digested with EcoRI and SalI and cloned into the EcoRI/SalI site of the pMal-c2X plasmid (Westburg B.V., Leusden, NL). Subsequently the DNA sequences coding for FNIII29 and FNIII29-30 of TNX were cloned into the EcoRI/SalI site of the pGex-4T1 plasmid (GE Healthcare Life Sciences, Diegem, BE). The sequence of the TNX domains was verified by dideoxy sequencing with a 3730 DNA analyzer (Applied Biosystems, Nieuwekerk a/d IJssel, NL). TNX fragments were obtained as maltose binding protein (MBP) fusion proteins using E.coli TOP10F' cells (Invitrogen) according to the manufacturer's instructions. MBP fusion proteins were isolated on amylose resin columns and analyzed for purity with SDS-PAGE. MBP fusion proteins were incubated with factor Xa (Westburg B.V.) in an attempt to remove the MBP tag from the fusion protein according to the manufacturer's instructions. FNIII29-30 without tag was obtained by proteolytic cleavage of FNIII29-30 from a glutathione- $S$-transferase (GST) fusion protein bound to a glutathionesepharose4B column (GE Healthcare Life Sciences) according to the manufacturer's instructions.

Proteins and antibodies

A $100 \mathrm{kDa}$ C-terminal recombinant TNX protein, containing six FNIII repeats and a fibrinogen domain, encoded by a $2.7 \mathrm{~kb}$ TNX sequence has previously been described by Tee et al. [19, 23]. MBP-FNIII2728, MBP-FNIII28-29, MBP-FNIII29, MBP-FNIII2930, MBP-FNIII30-31, MBP-FbgX and FNIII29-30 were obtained as described in the previous section.

Human Collagen type I from Chemicon International (Chemicon International, Temecula, CA, USA), human collagen type III and $\mathrm{V}$ from Rockland Inc (Tebu-bio, Heerhugowaard, NL). Bovine collagen type I (acid soluble) was obtained from BD biosciences (BD Biosciences, Alphen aan den Rijn, NL). Recombinant bovine tropoelastin (bTE) was a generous gift from Dr. R. Mecham (Washington University, St. Louis, MO, USA) [10]. Human recombinant fibrillin domains, Tb2cbEGF11, Tb3-cbFGF11, cbEGF11-14, cbEGF22-23, cbEGF28-30, tb6-cbEGF32, cbEGF32-36, tb7-cbEGF37, were generously donated by Dr. P.A. Handford (Oxford University, Oxford, UK) [7, 9, 11, 17, 24, 25]. BSA and gelatin were obtained from Sigma-Aldrich (Sigma-Aldrich Chemie B.V., Zwijndrecht, NL). BSA was used as a negative control. MBP was obtained from New England Biolabs (Westburg B.V.) and used as a negative control.

We used monoclonal antibodies specific for MBP (Westburg B.V) and a previously described guinea piganti-TNX serum [22] for detection of untagged $100 \mathrm{kDa}$ TNX and MBP-TNX-domains in our solidphase assays. Pre-immunization serum was used as a

Table 1 TNX domains and primers

\begin{tabular}{|c|c|c|}
\hline TNX domains & Forward primer & Reverse primer \\
\hline FNIII27-28 & 5'-GGAATTCGAGCTACCTCCCCAC-3' & 5'-CTCGTCGACTCACTGACCAGCAGGAGC-3' \\
\hline FNIII28-29 & 5'-CTGAATTCCCTGAAGAGCCCCGC-3' & 5'-TGGGTCGACTCAGCGGGCGGTTCCCTG-3' \\
\hline FNIII29 & 5'-TCAGAATTCTCAAGGCCCCGCCTG-3' & 5'-TGGGTCGACTCAGCGGGCGGTTCCCTG-3' \\
\hline FNIII29-30 & 5'-TCA GAATTCTCAAGGCCCCGCCTG-3' & 5'-GAAGTCGACTCAAGGCTCACTCTCСТC-3' \\
\hline FNIII30-31 & 5'-ACCEAATTCACCCTCAGCCCAGTT-3' & 5'-GTGTCGACTCAGATGCTGGCTGGGG-3' \\
\hline FbgX & 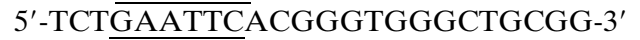 & 5'-GG $\overline{\overline{G T C G A C}} A G A G A G G T G G G C A G C A-3^{\prime}$ \\
\hline
\end{tabular}

The EcoRI and SalI restriction sites are underlined in the primer sequences. Each SalI site is preceded by a stop codon (TCA), except for the fibrinogen domain, which already contains a stop codon at the $3^{\prime}$ end of the sequence 
negative control [22] FNIII29-30 of TNX was detected with a new affinity purified polyclonal rabbit antiserum, raised against a MBP-FNIII29-30 antigen.

\section{Collagen fibrillogenesis assays}

Native bovine type I collagen (BD Biosciences, Alphen aan den Rijn, NL) $2.9 \mathrm{mg} / \mathrm{ml}$ in $0.012 \mathrm{~N} \mathrm{HCl}$, as provided by the manufacturer, was dissolved in PBS. MBP, BSA, MBP-FNIII29, FNIII29-30 and $100 \mathrm{kDa}$ TNX were added to samples in cuvettes after which the final volume was adjusted to $1 \mathrm{ml}$ of $0.4 \mathrm{mg} / \mathrm{ml}$ collagen type I with PBS and incubated at $30^{\circ} \mathrm{C}$. Turbidity change was measured by monitoring the change in absorbance at $400 \mathrm{~nm}$ in a UV-160A Shimadzu spectrophotometer (Shimadzu Benelux, Den Bosch, NL) at fixed intervals. Data points represent the mean of triplicate measurements.

Binding assays

Ninety-six well microtiter plates (Greiner Bio-One B.V., Alphen aan den Rijn, NL) were coated overnight at $4^{\circ} \mathrm{C}$ with ECM components diluted in PBS. All further incubation steps were performed at $37^{\circ} \mathrm{C}$. After each incubation step wells were washed with PBS containing $0.05 \%$ Tween-20 (T-PBS). Wells were saturated with $1 \%$ BSA in T-PBS for $2 \mathrm{~h}$ and then incubated with recombinant TNX domains for $1 \mathrm{~h}$. This was followed by incubation with antibodies against the recombinant TNX fragments for $1 \mathrm{~h}$. Thereafter, wells were incubated with biotinylated anti guinea pig (Vector Laboratories Inc., Burlingame, CA, USA), biotinylated anti rabbit (Vector Laboratories Inc.) or anti mouse IgG (vectastain kit, Brunschwig Chemie, Amsterdam, NL) for $1 \mathrm{~h}$ followed by a $45 \mathrm{~min}$ incubation with an avidin-biotin-horse radish peroxidase mixture (vectastain kit, Brunschwig Chemie). Bound peroxidase was detected with $o$-phenylenediamine dihydrochlorid (Perbio Science Nederland B.V., Etten-Leur, NL) and the absorbance read at $490 \mathrm{~nm}$. All antibodies and TNX proteins were diluted in T-PBS containing $0.1 \%$ BSA. PBS was substituted by tris buffered saline (TBS) $+5 \mathrm{mM}$ $\mathrm{Ca}_{2} \mathrm{Cl}_{2}$ in solid phase assays in which binding of TNX to fibrillin-1 domains was investigated for proper folding/stability of the fibrillin-1 domains [7, 21]. Assays were performed at least twice. BSA, MBP and pre immunization guinea pig serum were used as negative controls or baseline correction.

As an alternative to the binding assay in ELISA format we investigated binding of MBP-FNIII29 to collagen fibrils generated during the fibrillogenesis assay.
Briefly, various concentrations of MBP-FNIII29 were incubated overnight at $30^{\circ} \mathrm{C}$ in the presence of $0.1 \%$ BSA or collagen type I $(0.4 \mathrm{mg} / \mathrm{ml})+0.1 \%$ BSA. Thereafter, the insoluble collagen fibrils were pelleted by centrifugation. The concentration of MBP-FNIII29 in the supernatant was determined using an assay similar to a previous described one [22] in which, instead of guinea pig anti TNX antiserum, fusion protein was detected with monoclonal antibodies specific for MBP (Westburg B.V.).

\section{SDS-PAGE analysis}

Proteins were loaded onto 12\% Bis-Tris gels (Invitrogen) gel electrophoresis was carried out using the NuPAGE system according to the manufacturer's instructions (Invitrogen). To analyze protein purity gels were stained using coomassie brilliant blue R250 (Brunschwig).

\section{Results}

Binding of C-terminal $100 \mathrm{kDa}$ TNX to extracellular matrix components

We postulated that TNX might interact with components of elastic fibers or collagens directly because of the disturbed properties of collagens and elastic fibers in TNX deficient patients. As a first approach to identify extracellular ligands of TNX, we performed solid phase assays to test binding of a recombinant $\mathrm{C}$-terminal $100 \mathrm{kDa}$ TNX fragment $[19,23]$ (soluble phase) to ECM molecules (immobilized substrates). Figure 1 a shows that TNX binds dose dependently to human collagens type I, III and V. Binding to BSA, which served as a negative control, was absent. Figure $1 \mathrm{~b}$ compares the relative values of recombinant C-terminal $100 \mathrm{kDa}$ TNX binding to human fibrillar collagens, at the highest concentration of fusion protein compared to the control values of BSA. Figure 2 a shows the interaction of TNX with bovine collagen type I, bovine tropoelastin and denatured collagen type I (gelatin). TNX did not bind to denatured bovine collagen (gelatin). Figure $2 \mathrm{~b}$ compares the relative values of recombinant C-terminal $100 \mathrm{kDa}$ TNX binding to bovine tropoelastin, collagen type I and gelatin, at the highest concentration of fusion protein compared to the control values of BSA. Half maximal saturation, a measure for the affinity of the interaction, was reached at a concentration of soluble $100 \mathrm{kDa}$ TNX of $1.8 \times 10^{-8} \mathrm{M}$ for human collagen type I and $4.0 \times 10^{-8} \mathrm{M}$ for bovine collagen type I. Half maximal saturation was reached at 

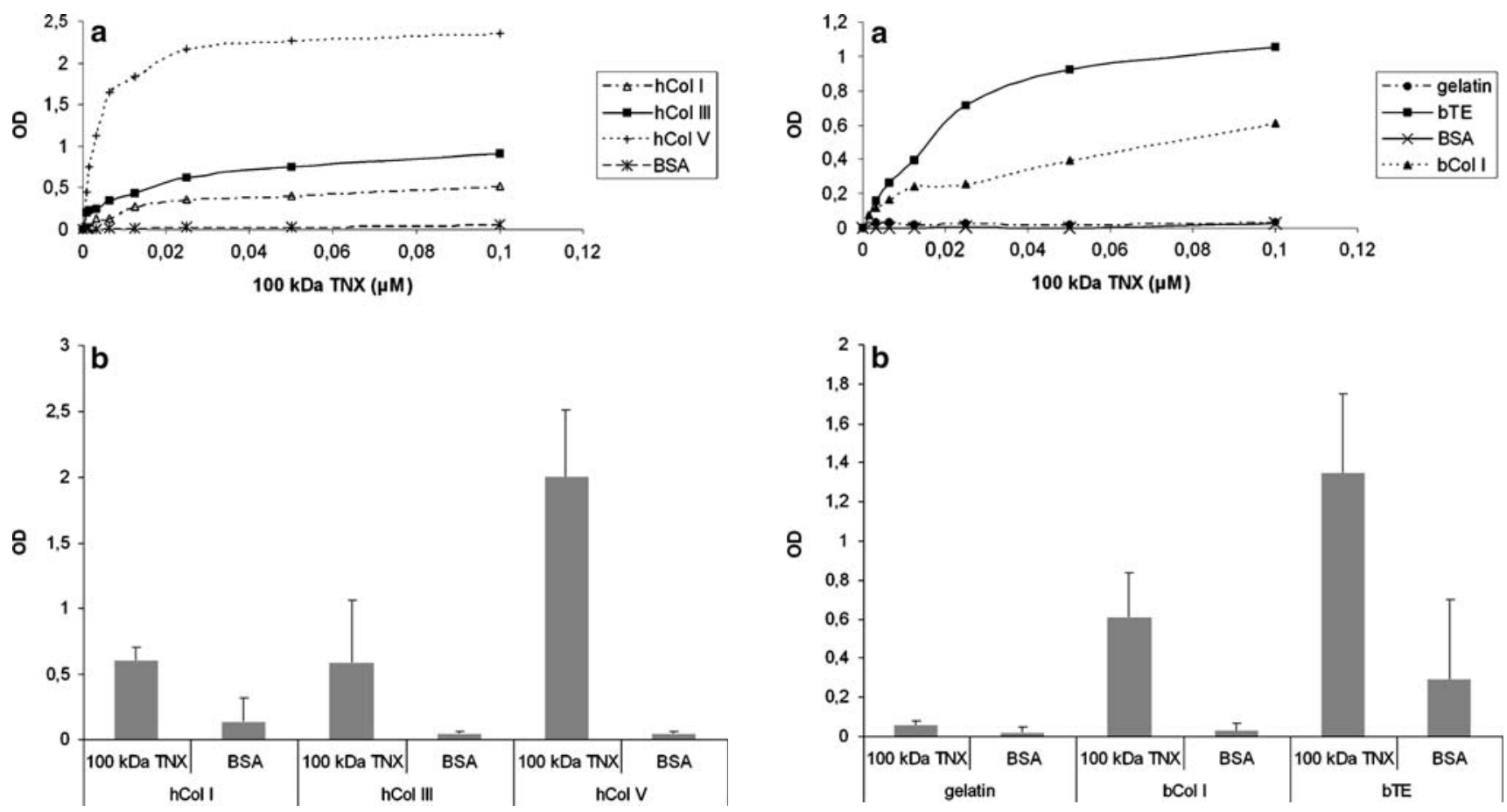

Fig. 1 Binding of recombinant C-terminal $100 \mathrm{kDa}$ TNX to human collagen type I, III and V. a TNX binds dose dependently to human collagens type I, III and V. Binding to BSA, which served as a negative control, was absent. b Comparisons of binding of recombinant C-terminal $100 \mathrm{kDa}$ TNX and BSA to collagens type I, III, and $\mathrm{V}$ at the highest concentration used $(10 \mu \mathrm{M})$

$1.1 \times 10^{-8} \mathrm{M}$ for human collagen type III, $0.8 \times 10^{-8} \mathrm{M}$ for human collagen type $\mathrm{V}$ and $1.8 \times 10^{-8} \mathrm{M}$ for tropoelastin. We did not observe binding to several domains of the elastic fiber protein fibrillin-1 (data not shown).

Binding of specific C-terminal TNX fragments to extracellular matrix components

To identify the sequences in TNX responsible for binding to the ECM components we subcloned and expressed a number of fragments from the C-terminal $100 \mathrm{kDa}$ TNX protein. Figure 3 gives a schematic overview of these fragments, and shows the purity of the fusion proteins used in this study. The domains studied here were produced as MBP-fusion proteins and included overlapping tandems of FNIII repeats and the FbgX domain.

All recombinant proteins containing the FNIII29 repeat showed dose dependent binding to collagen type I, III and V. As an example the binding curves for collagen type V are shown in Fig. 4a. Only FNIII29, FNIII28-29 and FNIII29-30 showed strong binding, whereas no binding was observed for repeats FNIII2728, FNIII30-31 and the FbgX domain which yielded a flat line as for the MBP and BSA control. For reasons

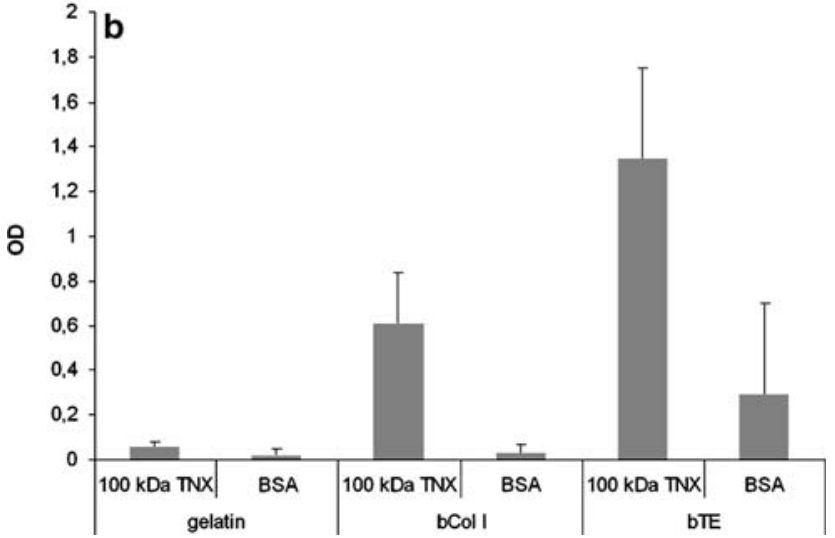

Fig. 2 Binding of recombinant C-terminal $100 \mathrm{kDa}$ TNX to bovine collagen type I and bTE. a TNX binds dose dependently to bTE and bovine collagen type I. No binding to denatured bovine collagen type I (gelatin) was observed (the gelatin signal coincides with the BSA baseline signal). b Comparison of binding of recombinant C-terminal $100 \mathrm{kDa}$ TNX and BSA to collagens type I, III and V at the highest concentration used $(10 \mu \mathrm{M})$

of clarity the curves for FNIII27-28, FNIII30-31, FbgX and BSA are not included in Fig. 4a because they coincide with MBP. Figure $4 \mathrm{~b}$ compares the relative values of FNIII29 and FNIII27-28 binding to fibrillar collagens, at the highest concentration of fusion protein compared to the control values of MBP.

Figure 5a depicts binding of TNX fragments to recombinant bTE. All fusion proteins containing FNIII29 were found to bind to TE, but in addition we found interaction with the FbgX domain, which was not the case for collagens. Recombinant MBP shows some non-specific binding to bTE but this is clearly less than FNIII29 and FbgX containing recombinant proteins. This non-specific binding was also observed for BSA, FNIII27-28 and FNIII30-31. The lines from these proteins coincide with the line of the MBP control, therefore they were omitted from Fig. 5a for reasons of clarity. Figure $5 \mathrm{~b}$ compares the relative values of FNIII29, FNIII27-28 and FbgX binding to recombinant TE, at the highest concentration of fusion protein compared to the control values of MBP.

We used TNX domains fused to MBP because this allows simple high yield purification and-importantly-it allows comparison and easy detection of bound fusion proteins by a single anti-MBP antibody. 
Fig. 3 Recombinant I'NX proteins used in this study. a Coomassie staining of recombinant proteins, lanes 1-5 MBP-FNIII repeats, lane $6 \mathrm{MBP}-\mathrm{FbgX}$ domain, lane 7 FNIII29-30 repeat without MBP fusion tag. b Schematic overview of recombinant proteins
MBP fusion proteins

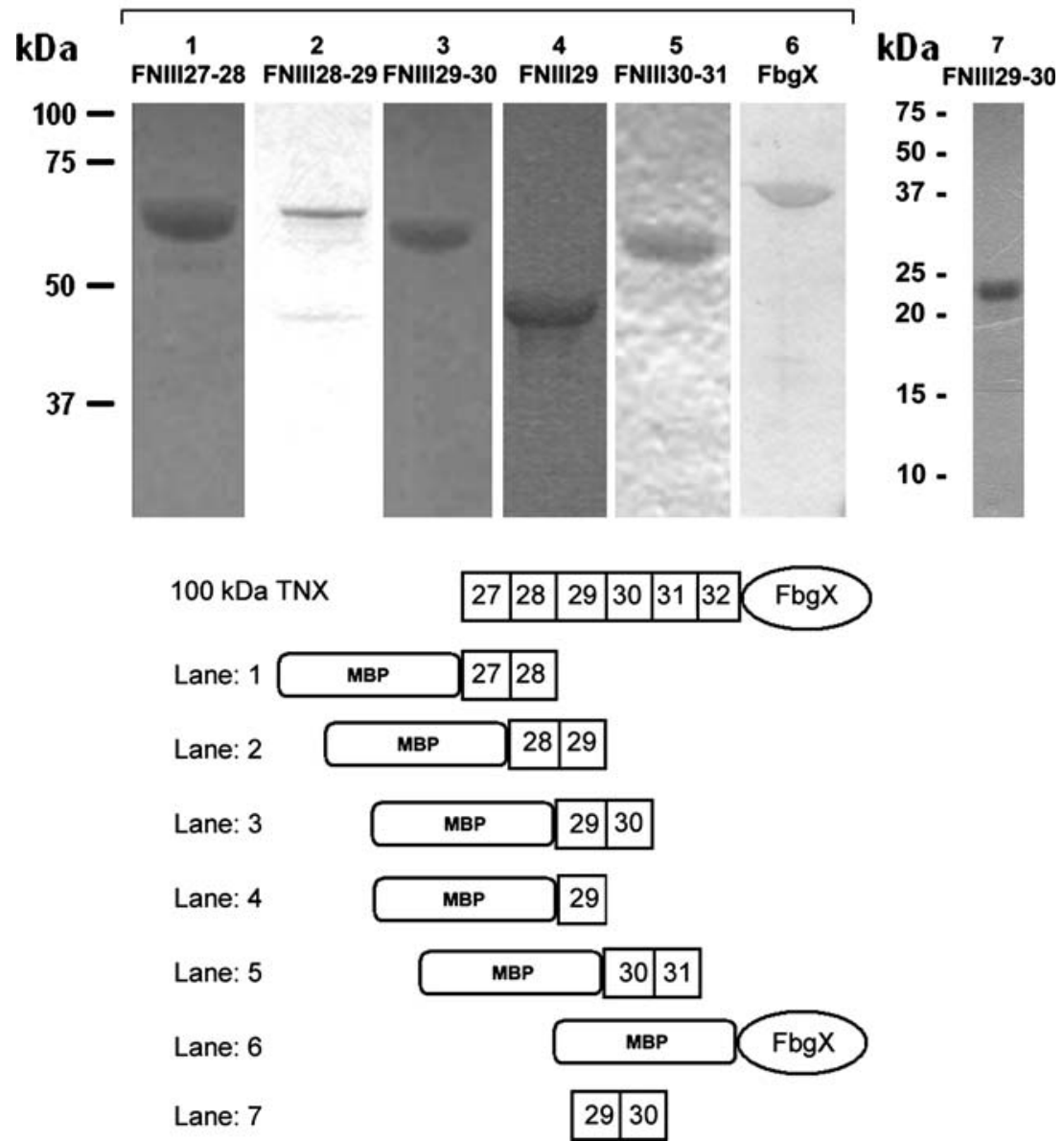

MBP alone was used as a control throughout the studies described above. In order to check if MBP fused to TNX domains would cause artificial binding of fusion proteins to ECM molecules we decided to test binding of a recombinant TNX fragment without MBP. Unfortunately, it was not possible to produce FNIII29 as a single repeat protein without fusion protein tag in either the pMal protein fusion or GST gene fusion system. We therefore produced a recombinant FNIII2930 repeat devoid of fusion tag and raised an antibody against FNIII29-30. Figure 6 shows that pure recombinant FNIII29-30 essentially has the same binding properties towards fibrillar collagens and TE as the MBPfusion protein, indicating that the interaction site resides in the TNX moiety (FNIII29) of the fusion proteins.

\section{Stimulation of fibrillogenesis by FNIII29}

To extend the observed binding properties of TNX FNIII repeats at the functional level, we investigated whether the collagen binding FNIII29 repeat of human TNX could modulate collagen fibrillogenesis. Using a turbidity assay (see Fig. 7a) we found an increase in the rate of fibril formation and a shortening of the lag phase in the presence of the FNIII29 repeat of TNX compared to the control proteins (BSA (not shown) and MBP) The fibrillogenesis rate in the lateral growth phase, characterized by fibril- and aggregate formation $[8,26]$, was significantly increased $(P<0.001)$ when $48 \mu \mathrm{g} / \mathrm{ml}$ MBP-FNIII29 was added. At a concentration of $24 \mu \mathrm{g} / \mathrm{ml}$, MBP-FNIII29 does not significantly influence the lateral growth phase, although it shortens the lag phase, characterized by linear growth of microfibrils and early fibril formation $[8,26]$, compared to controls. After $24 \mathrm{~h}$ all samples reached a similar maximal OD (data not shown). FNIII29-30 had only a minimal effect on collagen fibrillogenesis compared to MBP-FNIII29 and C-terminal $100 \mathrm{kDa}$ TNX showed no significant effect on either the lag- or lateral growth phase of collagen fibrillogenesis (data not shown).

The fibrillogenesis assay also provided us with an alternative method to investigate binding of TNX fragments to ECM molecules. To investigate the binding of MBP-FNIII29 to the collagen fibrils formed during the assay, we measured the amount of MBP-FNIII29 that 

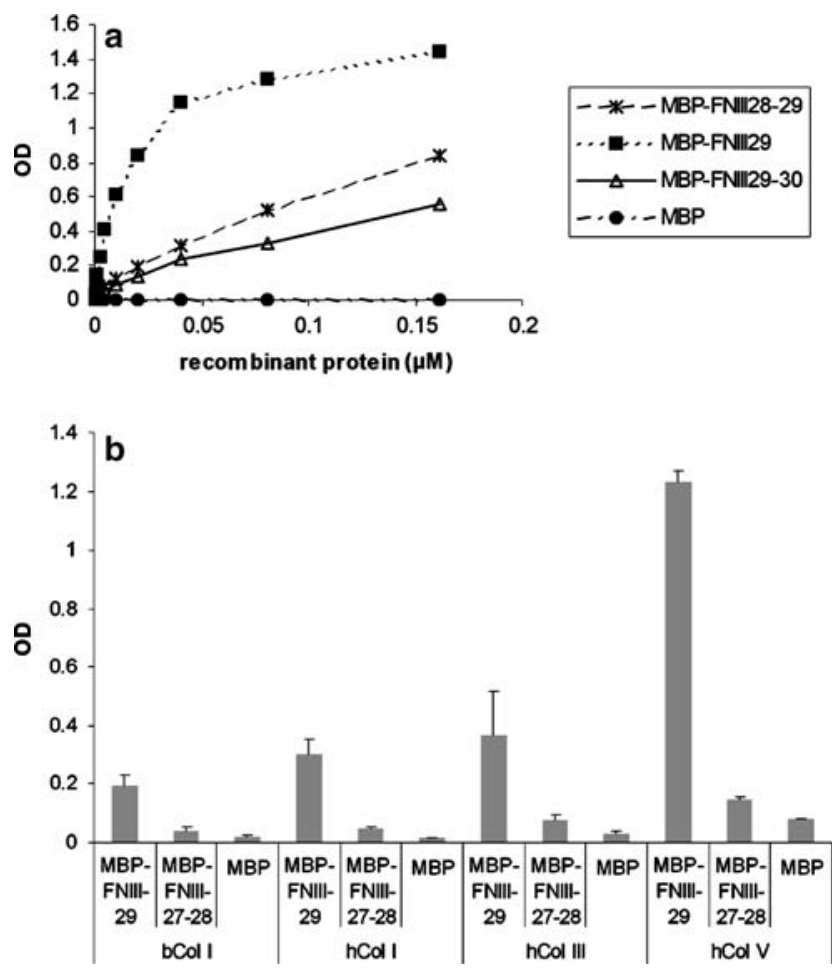

Fig. 4 Binding of TNX FNIII repeats to collagen type I, III an V. a Binding curves of recombinant TNX proteins containing FNIII29 show dose dependent binding to fibrillar collagens as shown here for human collagen type V. b Comparison of binding of MBP-FNIII29, MBP-FNIII27-28 and MBP to collagens type I, III and $\mathrm{V}$ at the highest concentration used $(16 \mu \mathrm{M})$

remained in the supernatant after pelleting of the insoluble collagen fibrils. As shown in Fig. 7b MBPFNIII29 was depleted from the soluble phase, indicating pull-down by the formation of collagen fibrils.

\section{Discussion}

We established a number of novel in vitro binding properties of human TNX that reside in the $100 \mathrm{kDa}$ $\mathrm{C}$-terminal portion. The binding properties of this fragment could be largely ascribed to the FbgX domain (tropoelastin binding) and the FNIII29 repeat, which was found to bind strongly to tropoelastin and collagen types I, III and V. Previous studies by others, using bovine and murine TNX have found binding to decorin [4] and collagen type I [18]. In the latter study, deletion of the EGF-like repeats or the FbgX domain did not abolish collagen binding, suggesting that binding of murine TNX resides somewhere in the FNIII repeats.

The binding to elastin was particularly interesting, as abnormal elastic fiber morphology is a characteristic feature of EDS patients with a complete TNX deficiency. We found binding of tropoelastin both to
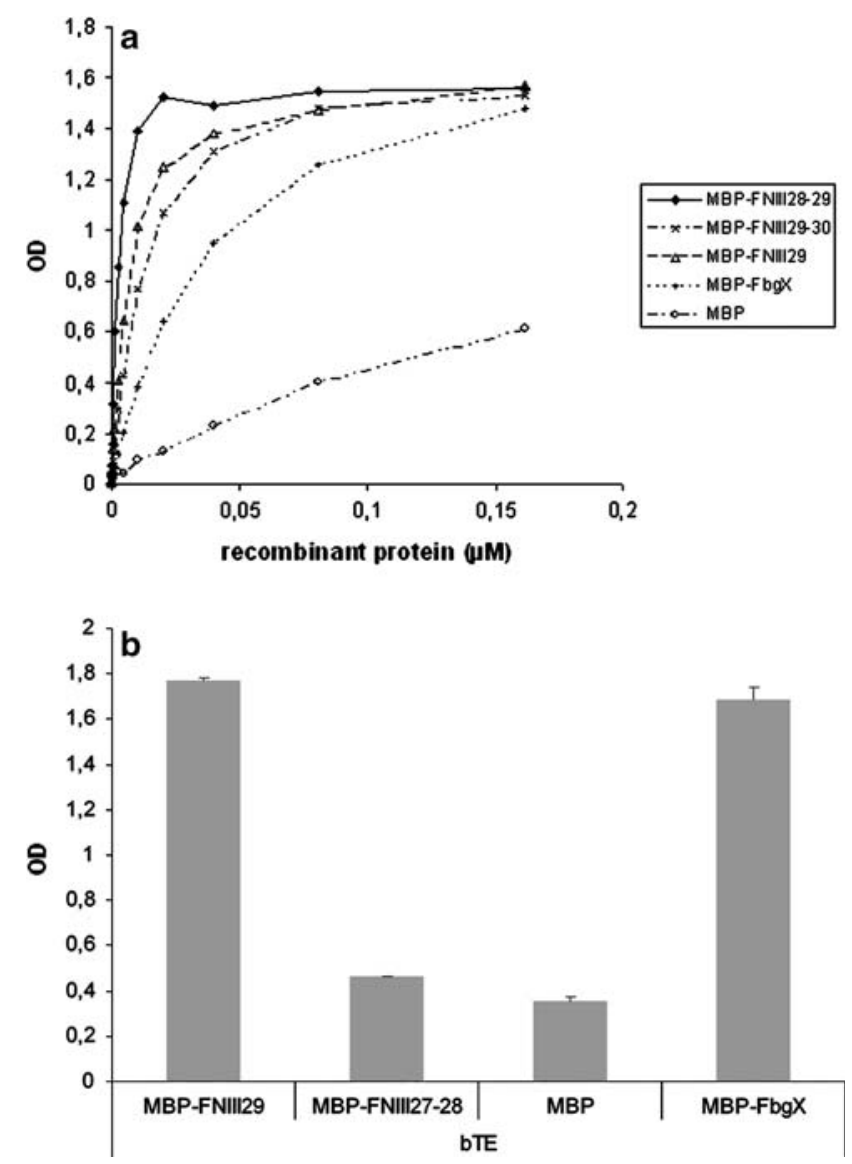

Fig. 5 Binding of TNX FNIII repeats and FbgX domain to TE. a Recombinant TNX fragments containing FNIII29 and the FbgX domain bind dose dependently to recombinant TE. The other investigated FNIII repeats of TNX do not show significant binding to bTE (not shown here). MBP alone shows non-specific binding which is significantly lower than the TNX containing fusion proteins. b Comparison of binding of MBP-FNIII29, MBPFNIII27-28, MBP-FbgX and MBP to recombinant TE at the highest concentration used $(16 \mu \mathrm{M})$

FNIII29 and FbgX. In contrast to FNIII29 the FbgX domain only binds to TE and not to other tested ECM proteins. The functional significance of this finding requires further investigation, but this interaction could be involved both in maturation or stability of the elastic fiber.

Dermal collagen deposition is reduced in TNX deficient patients, and their skin and other connective tissues is fragile as witnessed by easy bruising $[22,28]$. The skin strength in TNX deficient mice is markedly reduced, although the significance and magnitude of the reduction of collagen deposition is debated $[3,15$, 18]. In mice, the variation in collagen fibril diameter appears to be increased $[15,18]$. Speculatively, the assembly or stability of collagen fibrils could be disturbed in TNX deficient patients. We found that the FNIII29 domain of human TNX is able to accelerate 


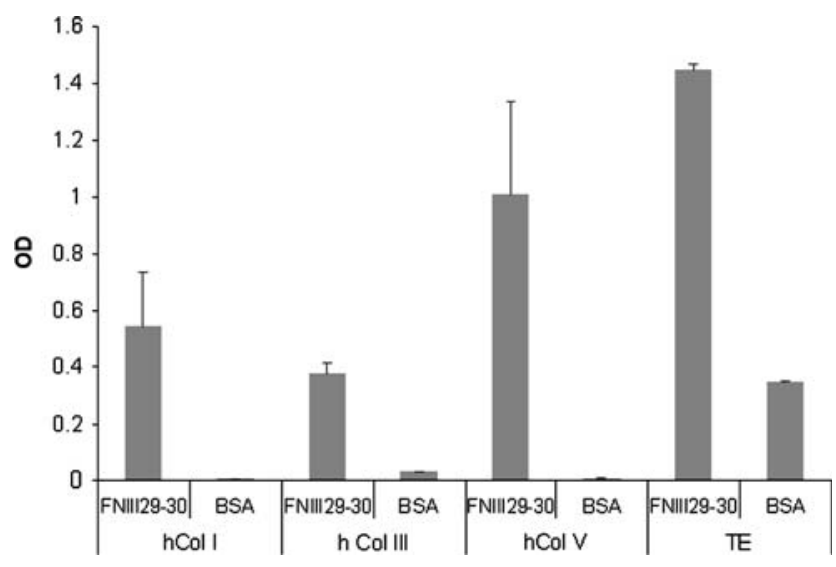

Fig. 6 Binding of FNIII29-30 to ECM proteins. Recombinant FNIII29-30 (without fusion tag) binds to collagen types I, III and $\mathrm{V}$ and to TE essentially in the same manner as the MBP fusion proteins containing FNIII29. BSA is used as a control for background staining $(7.5 \mu \mathrm{g} / \mathrm{ml})$

the collagen fibril formation in vitro. In contrast, however, we failed to detect a significant effect of the $100 \mathrm{kDa}$ TNX fragment on fibrillogenesis. This might possibly be due to conformational changes caused by folding of the $100 \mathrm{kDa}$ TNX in solution that precludes an effect on fibrillogenesis, although the $100 \mathrm{kDa}$ fragment binds to collagen type I. Alternatively, the observed effect of the TNX FNIII29 repeat on fibrillogenesis could be artificial, because it is not known whether it is accessible to collagen in the native $450 \mathrm{kDa}$ protein. Minamitani et al. found that a short isoform of murine TNX could accelerate collagen fibrillogenesis in an assay similar to the one we used [18]. Interestingly, they found that deletion of the EGF repeats or fibrinogen domain did not have an effect on binding of TNX to collagen although collagen fibrillogenesis kinetics were affected by deletion of these domains. Possibly conformational changes could account for this affect. Furthermore, we observed binding of the TNX FNIII29 repeat to the insoluble collagen fibrils formed during in vitro fibrillogenesis. This is in agreement with the observed binding properties of this domain in our solid phase assay in ELISA format. For fibrillar collagens type III and V we observed that preincubation of TNX FNIII29 with these collagens reduced the measured amount of TNX compared to controls in a previously described TNX ELISA [22] (data not shown). These findings are in accordance with the results from our solid phase binding assays. Clearly, the functional consequences of the interaction of TNX domains require further investigation. Ideally, the complete human $450 \mathrm{kDa}$ [1] or adrenal specific $74 \mathrm{kDa}$ [23] TNX form with correct glycosylation should be produced or purified, to provide definitive
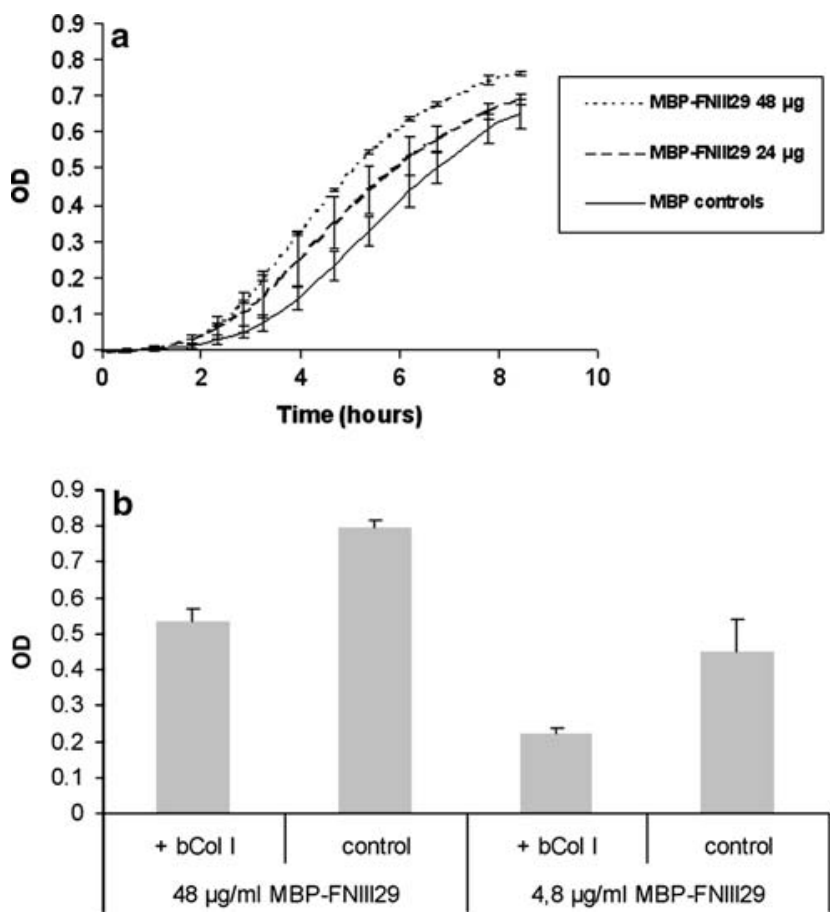

Fig. 7 TNX FNIII29 affects collagen fibrillogenesis. a TNX FNIII29 has a dose dependent effect on the fibrillogenesis rate of bovine collagen type I in a turbidimetric assay. At a concentration of $48 \mu \mathrm{g} / \mathrm{ml} \mathrm{MBP-FNIII29} \mathrm{the} \mathrm{fibrillogenesis} \mathrm{rate} \mathrm{in} \mathrm{the} \mathrm{lateral}$ growth phase is significantly increased. At a concentration of $24 \mu \mathrm{g} / \mathrm{ml}$ MBP-FNIII29 does not significantly influence the lateral growth phase, although at both 24 and $48 \mu \mathrm{g} / \mathrm{ml}$ the lag phase is shortened. MBP and BSA served as a negative control. The curves of BSA and $4.8 \mu \mathrm{g} / \mathrm{ml}$ MBP-FNIII29 overlapped with the MBP curve and have been omitted for reasons of clarity. b Comparison of TNX FNIII29 levels in the supernatant of pelleted insoluble collagen fibrils compared to controls. Differences of TNX FNIII29 concentration in the supernatant reflect the amount of TNX FNIII29 bound to the pelleted insoluble collagen fibrils

answers with respect to molecular interactions and effects on fibrillogenesis. On the other hand, the molecular diversity of TNX is far bigger than anticipated. We have found that various fragments of TNX, ranging from 70 to $450 \mathrm{kDa}$ are present in human serum (unpublished results). Whether these represent alternative splice forms or posttranslationally processed fragments is unknown. All of these TNX forms contain the FNIII29 repeat and it is possible that these shorter forms adopt a conformation in which FNIII29 is exposed to exert binding to ECM molecules and causes functional effects.

In conclusion, our study shows that domains of TNX directly interact with a number of ECM molecules that form a major part of the affected structures in the dermis of TNX deficient patients. Our data could serve as a starting point to investigate the function of individual domains in the context of the physiological TNX 
molecules, like the $450 \mathrm{kDa}$ tissue form or the various serum forms. Further investigation of the role of TNX in extracellular matrix assembly and stability will increase our knowledge of connective tissue biology and will offer a molecular explanation for the phenotype of TNX deficient patients.

Acknowledgments The authors are grateful to Dr P. Handford, Dr N. Grebenchtchikov and Dr R. Mecham for providing biological reagents used in this study.

\section{References}

1. Bristow J, Tee MK, Gitelman SE, Mellon SH, Miller WL (1993) Tenascin-X: a novel extracellular matrix protein encoded by the human XB gene overlapping P450c21B. J Cell Biol 122:265-278

2. Burch GH, Gong Y, Liu W, Dettman RW, Curry CJ, Smith L, Miller WL, Bristow J (1997) Tenascin-X deficiency is associated with Ehlers-Danlos syndrome (see comments). Nat Genet 17:104-108

3. Egging DF, van Vlijmen I, Starcher B, Gijsen Y, Zweers MC, Blankevoort L, Bristow J, Schalkwijk J (2006) Dermal connective tissue development in mice: an essential role for tenascin-X. Cell Tissue Res 323:465-474

4. Elefteriou F, Exposito J, Garrone R, Lethias C (2001) Binding of tenascin-X to decorin. FEBS Lett 495:44-47

5. Elefteriou F, Exposito JY, Garrone R, Lethias C (1997) Characterization of the bovine tenascin-X. J Biol Chem 272:22866-22874

6. Ikuta T, Sogawa N, Ariga H, Ikemura T, Matsumoto K (1998) Structural analysis of mouse tenascin-X: evolutionary aspects of reduplication of FNIII repeats in the tenascin gene family. Gene 217:1-13

7. Jensen SA, Corbett AR, Knott V, Redfield C, Handford PA (2005) $\mathrm{Ca} 2+$-dependent interface formation in fibrillin-1. J Biol Chem 280:14076-14084

8. Kadler KE, Holmes DF, Trotter JA, Chapman JA (1996) Collagen fibril formation. Biochem J 316(Pt1):1-11

9. Kettle S, Yuan X, Grundy G, Knott V, Downing AK, Handford PA (1999) Defective calcium binding to fibrillin-1: consequence of an N2144S change for fibrillin-1 structure and function. J Mol Biol 285:1277-1287

10. Kozel BA, Wachi H, Davis EC, Mecham RP (2003) Domains in tropoelastin that mediate elastin deposition in vitro and in vivo. J Biol Chem 278:18491-18498

11. Lee SS, Knott V, Jovanovic J, Harlos K, Grimes JM, Choulier L, Mardon HJ, Stuart DI, Handford PA (2004) Structure of the integrin binding fragment from fibrillin-1 gives new insights into microfibril organization. Structure (Camb) 12:717-729

12. Lethias C, Descollonges Y, Boutillon MM, Garrone R (1996) Flexilin: a new extracellular matrix glycoprotein localized on collagen fibrils. Matrix Biol 15:11-19

13. Lethias C, Elefteriou F, Parsiegla G, Exposito JY, Garrone R (2001) Identification and characterization of a conformational heparin-binding site involving two fibronectin type iii modules of bovine tenascin-X. J Biol Chem 276:16432-16438
14. Lindor NM, Bristow J (2005) Tenascin-X deficiency in autosomal recessive Ehlers-Danlos syndrome. Am J Med Genet A 135(1):75-80

15. Mao JR, Taylor G, Dean WB, Wagner DR, Afzal V, Lotz JC, Rubin EM, Bristow J (2002) Tenascin-X deficiency mimics Ehlers-Danlos syndrome in mice through alteration of collagen deposition. Nat Genet 30:421-425

16. Matsumoto K, Saga Y, Ikemura T, Sakakura T, Chiquet ER (1994) The distribution of tenascin-X is distinct and often reciprocal to that of tenascin-C. J Cell Biol 125:483-493

17. McGettrick AJ, Knott V, Willis A, Handford PA (2000) Molecular effects of calcium binding mutations in Marfan syndrome depend on domain context. Hum Mol Genet 9:1987-1994

18. Minamitani T, Ikuta T, Saito Y, Takebe G, Sato M, Sawa H, Nishimura T, Nakamura F, Takahashi K, Ariga H, Matsumoto K (2004) Modulation of collagen fibrillogenesis by tenascin-X and type VI collagen. Exp Cell Res 298:305-315

19. Morel Y, Bristow J, Gitelman SE, Miller WL (1989) Transcript encoded on the opposite strand of the human steroid 21-hydroxylase/complement component $\mathrm{C} 4$ gene locus. Proc Natl Acad Sci USA 86:6582-6586

20. Peeters ACTM, Kucharekova M, Timmermans J, van den Berkmortel FWPJ, Boers GH, Novakova IRO, Egging D, den Heijer M, Schalkwijk J (2004) A clinical and cardiovascular survey of Ehlers-Danlos syndrome patients with complete deficiency of tenascin-X. Neth J Med 62:23-25

21. Reinhardt DP, Mechling DE, Boswell BA, Keene DR, Sakai LY, Bachinger HP (1997) Calcium determines the shape of fibrillin. J Biol Chem 272:7368-7373

22. Schalkwijk J, Zweers MC, Steijlen PM, Dean WB, Taylor G, Van Vlijmen IM, van Haren B, Miller WL, Bristow J (2001) A recessive form of the Ehlers-Danlos syndrome caused by tenascin-X deficiency. N Engl J Med 345:1167-1175

23. Tee MK, Thomson AA, Bristow J, Miller WL (1995) Sequences promoting the transcription of the human XA gene overlapping P450c21A correctly predict the presence of a novel, adrenal-specific, truncated form of tenascin-X. Genomics 28:171-178

24. Whiteman P, Downing AK, Handford PA (1998) NMR analysis of cbEGF domains gives new insights into the structural consequences of a P1148A substitution in fibrillin-1. Protein Eng 11:957-959

25. Whiteman P, Smallridge RS, Knott V, Cordle JJ, Downing AK, Handford PA (2001) A G1127S change in calcium-binding epidermal growth factor-like domain 13 of human fibrillin-1 causes short range conformational effects. J Biol Chem 276:17156-17162

26. Williams BR, Gelman RA, Poppke DC, Piez KA (1978) Collagen fibril formation. Optimal in vitro conditions and preliminary kinetic results. J Biol Chem 253:6578-6585

27. Zweers MC, Bristow J, Steijlen PM, Dean WB, Hamel BC, Otero M, Kucharekova M, Boezeman JB, Schalkwijk J (2003) Haploinsufficiency of TNXB is associated with hypermobility type of Ehlers-Danlos syndrome. Am J Hum Genet 73:214217

28. Zweers MC, Vlijmen-Willems IM, Van Kuppevelt TH, Mecham RP, Steijlen PM, Bristow J, Schalkwijk J (2004) Deficiency of tenascin-X causes abnormalities in dermal elastic fiber morphology. J Invest Dermatol 122:885-891 